\title{
Deep Learning Based Prediction of Atrial Fibrillation Disease Progression with Endocardial Electrograms in a Canine Model
}

\author{
Bram Hunt $^{1}$, Eugene Kwan ${ }^{1}$, Mark McMillan ${ }^{2}$, Derek Dosdall ${ }^{1}$, Rob MacLeod ${ }^{1}$, Ravi Ranjan $^{1}$ \\ ${ }^{1}$ University of Utah, Salt Lake City, Utah, United States \\ ${ }^{2}$ Boston Scientific, Marlborough, Massachusetts, United States
}

\begin{abstract}
Objective: We sought to determine whether electrical patterns in endocardial wavefronts contained elements specific to atrial fibrillation $(A F)$ disease progression.

Methods: A canine paced model ( $n=7$, female mongrel, $29 \pm 2 \mathrm{~kg}$ ) of persistent $A F$ was endocardially mapped with a 64-electrode basket catheter during periods of $A F$ at 1 month, 3 month, and 6 months post-implant of stimulator. A 50-layer residual network was then trained to map halfsecond electrogram samples to their source timepoint.

Results: The trained network achieved final validation and testing accuracies of 51.6 and $48.5 \%$ respectively. Per class $\mathrm{F} 1$ scores were $24 \%$, 59\%, and 53\% for 1 month, 3 month, and 6 month inputs from the testing dataset.

Conclusion: Differentiation of AF based on its time progression was shown to be feasible with a deep learning method. This is promising for differentiating treatment based on disease progression though low accuracy with earlier timepoints may be an obstacle to identifying nascent AF.
\end{abstract}

\section{Introduction}

Atrial fibrillation (AF) is an arrhythmia coming from upper chambers of the heart and presents as rapid and uncoordinated contractions of the atria. AF is associated with increased risk of heart failure and stroke, and it affects $3-5 \%$ of the US population. ${ }^{[1]}$ Current therapies for AF, including pulmonary vein isolation and antiarrhythmic drug-based treatments, are associated with high recurrence rates, especially for patients with persistent $\mathrm{AF}^{\left[{ }^{[2]}\right.}$

The development of persistent AF remains poorly characterized and this presents an obstacle to differentiating therapies based on its progression over time. Because sinus rhythm becomes more difficult to maintain as AF develops, better characterization of differences between fibrillation over time may allow creation of superior treatments specific to disease progression by targeting time-specific electrical and substrate phenomena. ${ }^{[3]}$ However, it remains unclear if there exists differences in fibrillatory endocardial conduction over time. This work hypothesized that there are learnable elements in the electrical patterns of fibrillation which predict the length of time in diseased status and aimed to train a residual neural network to perform the analysis. The existence of learnable elements would be promising for treatments aiming to address the arrhythmia based on length of time in AF.

Deep residual networks have been shown to achieve high accuracy on image classification tasks through efficient backpropagation of learning gradients to upper layers of the network. ${ }^{[4]}$ In addition, the use of transfer learning with pretrained networks enables efficient training by exploiting the generalizability of weights used across image classification tasks. The combination of deep residual networks with transfer learning provides effective means for most image classifications.

\section{Methods}

A paced canine model $(\mathrm{n}=7$, female mongrel, $29 \pm 2 \mathrm{~kg}$, 12 months old) was used to model the progression of persistent AF. ${ }^{[5]}$ Neurostimulators were implanted with leads connected to cardiac tissue within the right atrial appendage of each animal. Pacing was performed at $50 \mathrm{~Hz}$ for 1 second every 4 seconds for 6 months. High-density endocardial voltage mapping was performed using the Boston Scientific Rythmia system in conjunction with a 64-electrode Orion catheter at a sampling rate of $955 \mathrm{~Hz}$. Mapping took place at 1 month, 3 months, and 6 months post implant during periods of sustained AF. These timepoints were the target classes for classification.

Mapping sites in the atria included the left and right appendages, posterior walls, superior walls, inferior walls, anterior walls, septal walls, free walls, and all veins. These sites were measured for 4 minutes each with the Orion catheter during fibrillation as verified through electrocardiography. The total number of measurement sites for all animals and timepoints was 558 .

Location and voltage data were exported directly from the Rythmia software after preprocessing. All further work was performed with custom MATLAB 2019b and Python 3 software. After exclusion of electrograms in poor contact based on signal quality, a total of 53,669 half second electrograms were isolated for deep learning. Training, validation, and testing split percentages were $55 \%, 15 \%$, and $30 \%$. In total, four animals were used for the training 
dataset, one for the validation dataset, and two for the testing dataset. Animals included in the testing dataset were excluded from the training and validation datasets, validation animals from training and testing datasets, and training animals from the testing and validation datasets. This ensured the generalizability of testing outcomes.

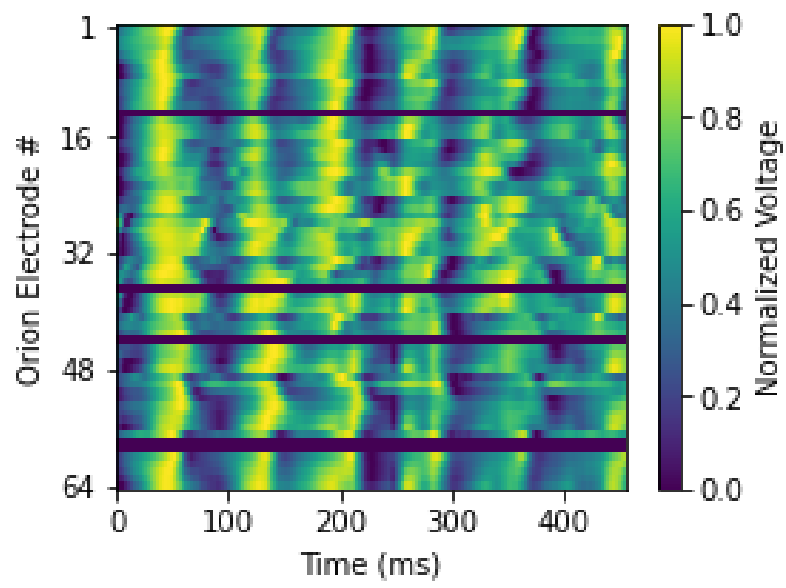

Figure 1. Example 64-electrode electrogram from training dataset. Voltage is normalized electrode-wise from 0 to 1 and smoothed with 10-pt time averaging. For training, between 0 and 10 electrodes were dropped from the electrogram and the electrodes were rotated randomly about the central axis of the catheter. This was done to reduce overfitting in the model.

A 50-layer ResNet pretrained on the ImageNet dataset was used to classify fibrillatory electrograms as originating from 1 month, 3 month, or 6 month measurements. ${ }^{[4]}$ The model and training algorithms were implemented in PyTorch, a Python 3 deep learning library. ${ }^{[6]}$ An example electrogram is shown in Fig. 1. The final fully connected layer of the network was reconfigured to produce 3 onehot coded classes, and input electrograms were tiled to a achieve an input size of $477 \times 64 \times 3$. The loss function used was binary cross entropy weighted to the proportion of each class in the training set with respect to the class with the highest proportion. Weighting values were 1.01, 1 , and 1.57 for 1 month, 3 month, and 6 month electrograms. This helped prevent overfitting to the most common class in training. Training was performed on a computer with a single GeForce RTX 2060 graphics card. The Adam optimization algorithm was used with an initial learning rate of 1e-4 and batch size of 25. ${ }^{[7]}$ The learning rate was halved every epoch. Validation accuracy was checked every 500 batches and at the end of every epoch to monitor network performance over time.

\section{Results}

The losses and accuracies from validation and training are shown in Fig. 2. Validation accuracy remained stable throughout training. After evaluation on the testing dataset, the network achieved a weighted testing accuracy of $49.2 \%$. This is an improvement over a theoretical random guess weighted accuracy of $33.3 \%$. Table I shows the confusion table for dataset classification. F1 scores were $24 \%, 59 \%$, and $53 \%$ for 1 month, 3 month, and 6 month inputs respectively.

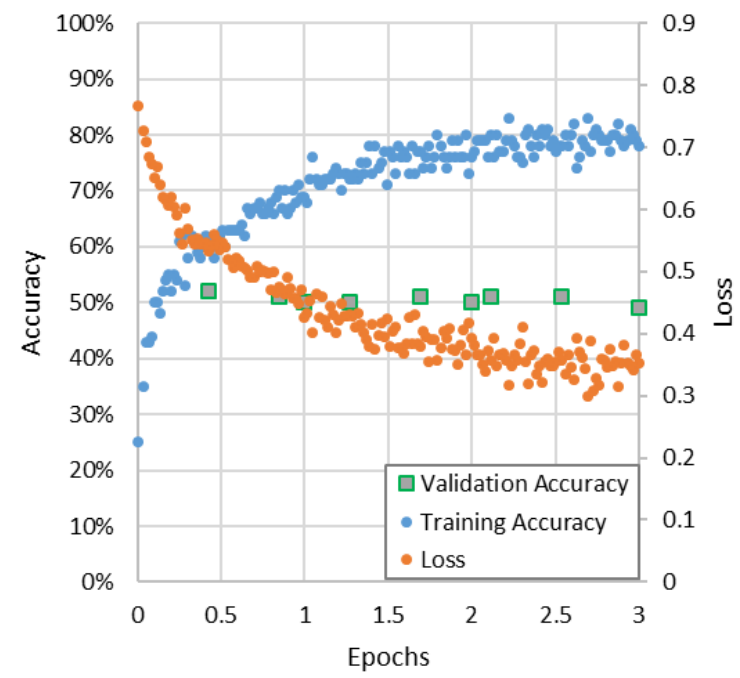

Figure 2. The network was trained over 3 epochs to a maximal validation accuracy of $51.6 \%$ and a training loss of 0.30 . Training was stopped after plateau in training accuracy.

Table 1. Confusion table for classification of the test dataset. F1 for 1 month samples is lower than those of other timepoints.

\begin{tabular}{llll}
\hline \multirow{2}{*}{ Truth } & \multicolumn{3}{c}{ Predicted } \\
\cline { 2 - 4 } & 1 Month & 3 Month & 6 Month \\
\hline $\begin{array}{l}\text { 1 Month } \\
(\mathrm{n}=5716)\end{array}$ & 941 & 3475 & 1300 \\
$\begin{array}{l}3 \text { Month } \\
(\mathrm{n}=5373)\end{array}$ & 509 & 4441 & 423 \\
$\begin{array}{l}6 \text { Month } \\
(\mathrm{n}=4803)\end{array}$ & 658 & 1814 & 2331 \\
\hline
\end{tabular}

\section{Conclusions}

This work successfully demonstrated the viability of using deep learning to predict the timecourse of AF from atrial electrograms during fibrillation. This has potential in allowing physicians to differentiate treatments based on long term AF duration by predicting patient disease progression. The existence of learnable electrical patterns implies there are unique electrical phenomena specific to developed AF, and that those phenomena, either in 
existence or extent, progress in a predictable manner as the development continues. These unique patterns may include patterns of conduction heterogeneity, action potential morphology, or particular conduction patterns such as focal activations and reentrant wavefronts. Targeting the sites of these indicators may improve therapies. The F1 score for 1 month electrograms in the testing dataset was low compared to the other classes. This may be improved with alternate training methods and models. Additionally, this could imply that AF which is early in development may not be readily distinguishable from more developed fibrillation. This would be explained by early fibrillation only containing electrical features which are present in other classes.

This study is limited in that the paced model of AF may have developmental differences from human AF. Additionally, the range of timepoints measured may not represent the entire meaningful range of AF progression. Future work should investigate a larger timepoint range as well as explore viability with clinical electrograms. Other network architectures or conventional algorithms may be able to improve on the present classification task.

\section{References}

[1] Y. Miyasaka. "Secular trends in incidence of atrial fibrillation in Olmsted County, Minnesota, 1980 to 2000, and implications on the projections for future prevalence," (vol 114, pg 119, 2006). Circulation, vol. 114, no. 2, pp 119-125, Jul. 2006 .
[2] E. Balk, A. Garlitski, A. Alsheikh-Ali, T Terasawa, M. Chung, S. Ip, "Predictors of atrial fibrillation recurrence after radiofrequency catheter ablation: a systematic review," $J$. Cardiovasc. Electrophysiol., vol. 21, no. 11, pp. 1208-1216, Nov. 2010.

[3] M. Frick, V. Frykman, M. Jensen-Urstad, J. Östergren, "Factors predicting success rate and recurrence of atrial fibrillation after first electrical cardioversion in patients with persistent atrial fibrillation," Clin. Cardiol., vol. 24, no. 3, pp. 238-244, Mar. 2001.

[4] K. He, X. Zhang, S. Ren, J. Sun, "Deep residual learning for image recognition," Proc. IEEE Comput. Soc. Conf. Comput. Vis. Pattern Recognit., 1512.03385, Dec. 2015.

[5] C. Morillo, G. Klein, D. Jones, C. Guiraudon, "Chronic rapid atrial pacing: structural, functional, and electrophysiological characteristics of a new model of sustained atrial fibrillation," Circulation, vol. 91, no. 5, pp. 1588-1595, Mar. 1995.

[6] A. Paszke, S. Gross, F. Massa, A. Lerer, J. Bradbury, G. Chanan, T. Killeen, Z. Lin, N. Gimelshein, L. Antiga, A. Desmaison, "Pytorch: An imperative style, highperformance deep learning library", Adv. Neural Inf. Process Syst., pp. 8026-8037, 2019.

[7] D. Kingma, J. Ba, "Adam: A method for stochastic optimization, " arXiv, 1412.6980, Dec. 2014.

Address for correspondence:

Bram Hunt

Nora Eccles Harrison CVRTI

95 S 2000 E, Salt Lake City, UT 84112

bram.hunt@utah.edu 